that $a \mu_{r m g} \geqq 1$, where $r^{m g}$ is the representative element of any class $\mathfrak{S}^{m} \theta$ to which $p$ belongs. Using such a function $\mu$ in the condition $(1 b)$ on the developmental system and noting that the sequence $\left\{\mu_{0 p_{2 n_{k}}}\right\}$ is bounded, we see that for every $e$ there is an $m_{e}$ such that for $m \geqq m_{e}$ and for every $k$ the value $\theta_{p_{2 n_{k}}}{ }^{m}$ does not exceed $e$. But this affords a contradiction to the conclusion reached above that

$$
L_{m}\left(L_{k} \theta_{p_{2 n_{k}}{ }^{m}}\right)=1 \text {. }
$$

Therefore the hypothesis that $p$ is not a limit of the sequence $\left\{p_{2 n}\right\}$ is contrary to fact.

These considerations may be extended to the infinite developments of Chittenden.*

Adelbert College,

WESTERN Reserve UNIVERSITY.

\title{
ON THE ADJOINT OF A CERTAIN MIXED EQUATION.
}

BY DR. R. F. BORDEN.

(Read before the American Mathematical Society December 30, 1919.)

ConsIDER a function of the form

(1) $F\{f(x)\}=\Delta f^{\prime}(x)+a(x) f^{\prime}(x)+b(x) \Delta f(x)+c(x) f(x)$,

where $a(x), b(x)$, and $c(x)$ are analytic functions of $x$, also $\Delta f(x)=f(x+1)-f(x)$, and $f^{\prime}(x)=(d / d x) f(x)$. We will say that $G\{g(x)\}$ is the adjoint of $F\{f(x)\}$ if $G\{g(x)\}=0$ is the condition that

$$
\int \Sigma g(x) F\{f(x)\} d x=\Sigma M(x)+\int N(x) d x,
$$

where $\Sigma$ denotes an inverse of $\Delta$.

This condition (2) is satisfied if

$$
g(x) F\{f(x)\}=\frac{d}{d x} M(x) d x+\Delta N(x),
$$

* Cf. "Infinite developments and the composition property $\left(K_{12} B_{1}\right)_{*}$ in general analysis," by E. W. Chittenden, Rendiconti del Circolo Matem. di Palermo, vol. 39 (1915), p. 21 , $\$ \S 19$ and 21 . 
where $M(x)$ and $N(x)$ are of the form

$$
\begin{aligned}
& M(x)=A(x) \Delta f(x)+B(x) f(x), \\
& N(x)=C(x) f^{\prime}(x)+D(x) f(x) .
\end{aligned}
$$

Equating corresponding coefficients in the two members of (3), we have

$$
\begin{aligned}
& g(x)=A(x)+C(x), \\
& a(x) g(x)=B^{\prime}(x), \\
& b(x) g(x)=A^{\prime}(x)+D(x), \\
& c(x) g(x)=D^{\prime}(x), \\
& \Delta C(x)=\Delta D(x)=0 .
\end{aligned}
$$

From (5) we have

$$
\begin{aligned}
\Delta g^{\prime}(x)-a(x) g^{\prime}(x)- & b(x+1) \Delta g(x) \\
+ & {\left[c(x)-a^{\prime}(x)-\Delta b(x)\right] g(x)=0, }
\end{aligned}
$$

which we will call the expression adjoint to the expression (1).* The equations formed by equating (1) and (6) to zero, we will call adjoint equations.

The formal mode of obtaining the coefficients of the adjoint gives (1) as the adjoint to (6) only when $\Delta b(x)=0$. However this difficulty may be avoided, for $b(x)$ may be taken identically zero without loss of generality, since (1) is transformed by the substitution

$$
f(x)=e^{-\int_{x_{0}}^{x} b(x) d x} h(x)
$$

into an expression having no term in $\Delta h(x)$. Accordingly in what follows we will take $b(x)$ as zero when discussing the adjoint.

We will now state a few facts about the equation $\dagger$

$$
\Delta f^{\prime}(x)+a(x) f^{\prime}(x)+b(x) \Delta f(x)+c(x) f(x)=0,
$$

where $a(x)$ is not identically one.

* Other forms of adjoint expressions may be found to satisfy (3), but among other advantages (6) affords in what follows the closest analogy to the theory of adjoint partial differential equations. See, for instance, Forsyth: Theory of Differential Equations, Vol. 6, p. 112.

† First studied by Poisson: Journal de l'Ecole Polytechnique, vol. 6. (1806), pp. 127-141. Its properties here listed are developed in my thesis which is to appear in the Amer. Jour. of Mathematics. 
(i) The equation (7) has under the group of transformations $f(x)=v(x) g(x)$ two fundamental invariants

and

$$
I(x)=\frac{c(x)-[a(x)-1] b(x)-a^{\prime}(x)}{a(x)-1}
$$

$$
J(x)=\frac{c(x)-[a(x)-1] b(x-1)}{a(x)-1} .
$$

(ii) When either $I(x)=0$ or $J(x)=0$, the equation may be solved in finite form by the standard methods of solving differential and difference equations, the two processes being separable.

(iii) If $n$ successive applications of the transformation

$$
f_{S_{1}}(x)=f(x+1)+[a(x)-1] f(x)
$$

or of the transformation

$$
f_{r_{1}}(x)=f^{\prime}(x)+b(x-1) f(x)
$$

result in an equation with a vanishing invariant, and if $n$ is the least integer for which this is true, then the equation is of rank $n+1$ with respect to the transformation.

(iv) If $I_{S_{m}}$ and $J_{S_{m}}$ are the invariants of the $m$ th transformed equation under $(S)$, and if $I_{T_{m}}$ and $J_{T_{m}}$ are the invariants of the equation resulting from $m$ applications of $I$, then

$$
J S_{n}^{S}(x)=I_{S_{n-1}}(x) \text { and } I_{T_{n}}(x)=J_{T_{n-1}}(x) .
$$

(v) The equation obtained by applying $(S)$ and $(T)$ in the order named, has the same invariants as has the equation obtained by applying first $(T)$ and then $(S)$.

The adjoint to

$$
\Delta f^{\prime}(x)+a(x) f^{\prime}(x)+c(x) f(x)=0
$$

$$
\Delta g^{\prime}(x)-a(x) g^{\prime}(x)+\left[c(x)-a^{\prime}(x)\right] g(x)=0,
$$

which has the invariants*

$$
i(x)=\frac{c(x)-a^{\prime}(x)}{-a(x)+1}+\frac{a^{\prime}(x)}{-a(x)+1}=\frac{c(x)}{-a(x)+1}=-J(x)
$$

* The discussion holds only when $a(x) \neq 1$. 
and

$$
j(x)=\frac{c(x)-a^{\prime}(x)}{-a(x)+1}=-I(x) .
$$

Hence the adjoint equation has the same set of invariants except for order as has the original equation. Consequently if one of two adjoint equations of the form (8) can be solved in finite form on account of a vanishing invariant, the same is true of the other.

Denote equation (8) by $F=0$ and its $n$th transform by $F_{S_{n}}=0$ or by $F_{T_{n}}=0$ according as $(S)$ or $(T)$ is used. Using (iv) and (v) we can express the invariants of the transformed equations as follows, each pair of invariants being written under the corresponding equation:

$$
\begin{aligned}
& F_{T_{n}}=0, \cdots, F_{T_{1}}=0, F=0, F_{S_{1}}=0, \cdots, F_{S_{n}}=0, \\
& I_{T_{n}}, \quad \ldots, I_{T_{1}}, \quad I, \quad I_{S_{1}}, \quad \ldots, I_{S_{n}}, \\
& I_{I_{n+1}}, \quad \ldots, I_{T_{2}}, \quad I_{T_{1}}, \quad I, \quad \ldots, I_{S_{n-1}} .
\end{aligned}
$$

Similarly we have for the adjoint equation $G=0$, the set

$$
\begin{aligned}
& G_{T_{n}}=0, \cdots, G_{T_{1}}=0, G=0, G_{S_{1}}=0, \cdots, G_{S_{n}}=0, \\
& I_{S_{n-1}}, \quad \ldots, I, \quad I_{T_{1}}, \quad I_{T_{2}}, \quad \ldots, I_{T_{n+1}}, \\
& I_{S_{n}}, \quad \cdots, I_{S_{1}}, \quad I, \quad I_{T_{1}}, \quad \ldots, I_{T_{n}},
\end{aligned}
$$

which differs from the set for $F=0$ and its transforms only in order.

From the two sets we see that, if an equation of the form (8) is of finite rank with respect to either $(S)$ or $(T)$, its adjoint is of the same rank with respect to the other transformation. Also if such an equation is of doubly finite rank, its adjoint is of doubly finite rank with the same index.

The equation

$$
\Delta f^{\prime}(x)+c(x) f(x)=0
$$

is self-adjoint. Its invariants are equal and have the value $-c(x)$. Unlike the corresponding partial differential equation, (10) is not a canonical form for equations with equal invariants. A necessary and sufficient set of conditions that (7) shall have equal invariants is

$$
a^{\prime}(x)=\Delta b(x)=0 .
$$


Not all equations of that form are reducible to the form (10); for example

$$
\Delta f^{\prime}(x)+2 f^{\prime}(x)+c(x) f(x)=0
$$

is not. However, equations with equal invariants which are not self-adjoint do not seem to be of great interest.

From the preceding theorem it follows that if a self-adjoint equation of form (10) is of finite rank with respect to one of the transformations $(S)$ or $(T)$, it is of the same rank with respect to the other.

Using the formula*

$$
\begin{aligned}
I_{S_{n}}(x)=I(x) & +\sum_{1}^{n-1}[I(x+k)-J(x+k)] \\
& -\Delta \frac{d}{d x} \log \left[\prod_{k=0}^{n-1} I(x+k) \prod_{k=0}^{n-2} I_{S_{1}}(x+k) \cdots I_{S_{n-1}}(x)\right]
\end{aligned}
$$

and noting that for the self-adjoint case

we have

$$
I(x)=J(x)=-c(x)
$$

$-c(x)=\Delta \frac{d}{d x} \log \left[\prod_{k=0}^{n-1}(-c(x+k)) \prod_{k=0}^{n-2} I_{S_{1}}(x+k) \cdots I_{S_{n-1}}(x)\right]$

as a necessary and sufficient condition that the self-adjoint equation (10) be of rank $n+1$ with respect to each of the transformations $(S)$ and $(T)$.

UNIVERSITY OF ILLINOIS.

\section{THE SECOND VOLUME OF VEBLEN AND YOUNG'S} PROJECTIVE GEOMETRY.

Projective Geometry. By Oswald Veblen and J. W. Young. Boston, Ginn and Company; Vol. 2, by Oswald Veblen, 1918. $12+511$ pages.

IN volume I, Veblen and Young were concerned particularly with those theorems of projective geometry which can be proved on the basis of their assumptions $A$ of alignment, assumptions $E$ of extension, and an assumption $P$ of pro-

\footnotetext{
* Formula (8) in my thesis, l.c.
} 